\title{
Towards Forming Communities Using Wearable Musical Instruments
}

\author{
Yukio TADA*, Kazushi NISHIMOTO ${ }^{* * * * * *}$, Tadao MAEKAWA*, \\ Romain Rouve", Kenji MASE* and Ryohei NAKATSU* \\ "ATR Media Integration and Communications Research Laboratories \\ ** Japan Advanced Institute of Science and Technology, Hokuriku \\ **** PRESTO, Japan Science and Technology Corporation \\ \{tada, knishi, maekawa, rouve, mase, nakatsu\}@mic.atr.co.jp
}

\begin{abstract}
In this paper, we describe the design and applications of "CosTune" (costume + tune), which is a novel wearable musical instrument. A CosTune is not only a personal wearable musical instrument but also is equipped with wireless communications functions to communicate with other CosTunes. A prototype system is also described. Music is a powerful communications medium, therefore, we designed CosTune as a communications tool. CosTune users can make collaborative compositions and perform ad hoc sessions with others who share similar musical tastes. Thus, CosTune may foster a novel musical culture as well as support the formation of communities mediated by music.
\end{abstract}

\section{Introduction}

In this paper, we describe a wearable musical instruments called "CosTune" (costume + tune) that is equipped with wireless communications functions. We discuss how CosTunes can help to enhance communications and to form communities in the real world.

Music plays an essential role as a communications medium. For example, members of a jazz band communicate with each other by performing music, and the band conveys a certain impression to the audience by their music. Another example is when we travel to a foreign country; we can communicate with local inhabitants by singing with them at a public house, dancing to music at a festival and so on, even if we cannot understand the language of the country. Thus, music is essentially a powerful communications medium as well as a universal language.

Up to now, however, music's characteristic as a communications medium has been neglected in the design of musical instruments. There are two problems to use ordinary musical instruments as communications tools. The first problem is that ordinary musical instruments are basically designed under the assumption that they will be used in a very restricted area, e.g., on a stage. Thus, portability is not considered in their design, and most instruments are too big and heavy to be carried. The second problem is that sound is the only available vehicle for users of ordinary musical instruments to communicate with others. It is usually difficult to convey complex information only by sound. It is also difficult to concurrently perform different pieces of music at the same place. Besides, the output sound troubles the unrelated people.

Several wearable musical instruments have been developed, e.g., YAMAHA MIBURI ${ }^{\mathrm{TM}}$ and BODYCODER [1], although their use is still restricted to on a stage. The Musical Jacket [2] developed at Media Lab., MIT, achieves true portability. However, the authors see it as a simple extension of ordinary musical instruments; sound is still the only vehicle for communications and the aspect of communications tool is not apparent in its design.

We designed "CosTune" to be a communications tool rather than a simple musical instrument. The most significant feature of CosTunes is that they are equipped with wireless network functions. This allows users to communicate with anyone, anytime, and anywhere by means of music in an ad hoc manner without scattering sound. We think that this feature makes CosTune a supporting tool for forming communities in the real world.

This paper is organized as follows: Section 2 describes the conceptual design of the CosTune system. Section 3 gives two interesting applications of the system and a skeleton of protocols for the applications. It also discusses how the system works as a communication tool. Section 4 describes a prototype system that was demonstrated at ATR exposition 2000. Section 5 discusses the differences between the CosTune and several musical performance 
systems over the Internet from the viewpoint of networked musical instruments. Section 6 is a brief conclusion.

\section{Conceptual design of system}

In this section, the design of the entire system is described. The system consists of CosTunes and servers. A CosTune is a portable musical instrument that can be worn by a user. A server is located somewhere in town and communicates with CosTunes that are passing by the server.

\subsection{The CosTune}

The CosTune consists of a wearable input device and a portable control unit (see Figure 1). The wearable input device is a cloth (e.g., jacket, pants and gloves) on which a number of tactile sensors that correspond to, for instance, keys of a piano are mounted. By manipulating the sensors, the user can play a musical performance. The portable control unit is equipped with an $\mathrm{A} / \mathrm{D}$ converter, a tone generator, a wireless communication component, a phrase storage unit and a sequencer.

The output signals from the sensors on the wearable input device are input to the $\mathrm{A} / \mathrm{D}$ converter that converts input analog signals into MIDI data. Currently, the mapping between a sensor and output MIDI data is stationary. However, variable mapping based on, for instance, "fixed note-function mapping concept" [3] is also useful to support novice players, in particular. The output MIDI data from the A/D converter and the sequencer that plays back stored phrases in the phrase storage unit are input to the tone generator. The output sound signals from the tone generator are input to the headphone. In the meanwhile, if the user wishes, the performed phrases, i.e., the output data from the A/D converter, are stored in the phrase storage.

The wireless communication component transmits as well as receives "phrase packets" (described in 2.3.) to and from other CosTunes and the servers. The phrase data as MIDI data that are obtained from the received phrase packets are also input to the tone generator as well as, if the user wishes, are stored in the phrase storage.

\subsection{The server}

The servers are located in places in towns like base stations of the cellular phone. The constituent of a server is almost same as the portable control unit of the CosTune, but the $A / D$ converter and the tone generator are not necessary (see Figure 2). The role of the server is to store sets of musical phrase data and to exchange them with the CosTunes. Therefore, the phrase storage of the server should be larger and more intelligent than that of the CosTune.

\subsection{Phrase packet}

The CosTunes and the servers exchange phrase packets. One phrase packet includes the following items:

- Phrase data

- $\quad$ Length of the phrase data

- Attributes of the phrase data, e.g., rhythm, tempo, timbre, a musical genre, a part in the musical structure

- An owner's profile, e.g., owner ID, age, sex, musical preferences and a place of residence.

Where, a phrase is a certain length of song component, which can be divided in time and/or instrumental type or role. Conversely, a song is defined as an organized set of phrases. Phrase data are sequences of symbols representing notes that the phrase consists of. MIDI format is usually employed to describe phrase data.

The relations among the musical genre, the part in the musical structure, and the timbre should be defined beforehand and shared by all CosTunes and servers. So, when a CosTune is going to exchange a phrase with another CosTune or with a server, one adequate phrase is chosen according to the relation. If a user indicates one

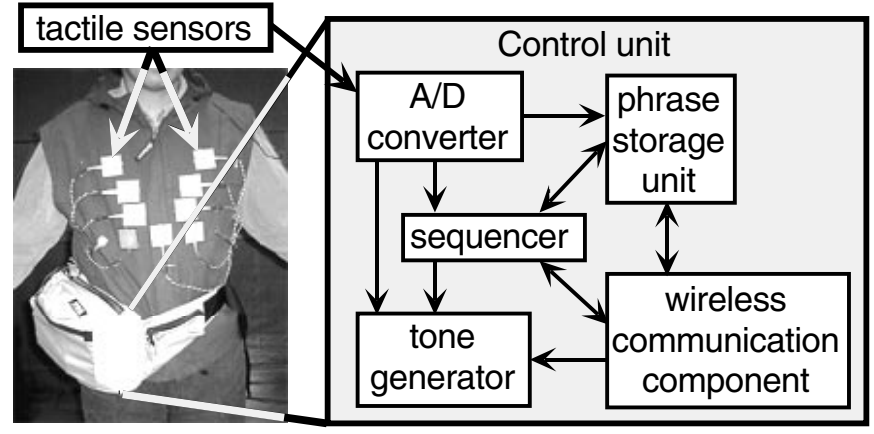

Figure 1 Components of the CosTune

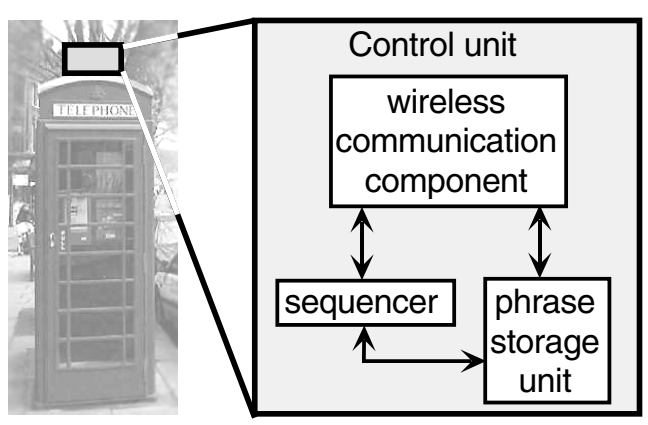

Figure 2 Components of the server 


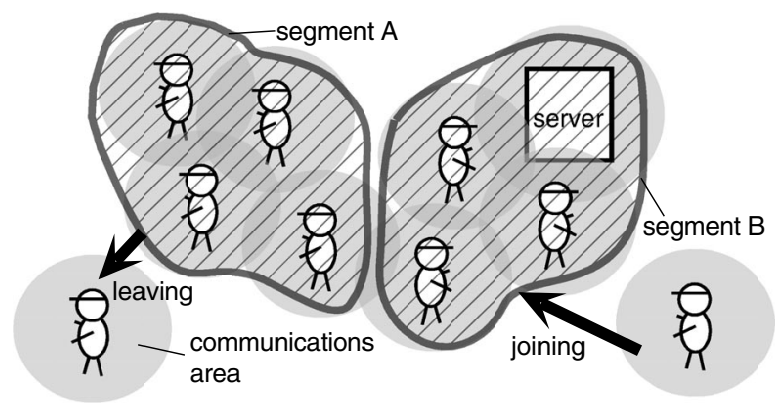

Figure 3 An example of an ad hoc network

specific genre and one specific part, his/her CosTune can collect all parts of the genre except for the indicated part, assemble them simultaneously, and form the accompanying data of a song for him/her. As a result, so-called "minus-one" data or "karaoke" data can be adaptively obtained.

\subsection{Requirements of ad hoc networking}

The ad hoc networking function is very important for the CosTune system. Figure 3 illustrates an example of an ad hoc network where two network segments already exist, i.e., segment A with four CosTunes and segment B with three CosTunes and a server.

Within a certain segment, all CosTunes and servers must be able to bi-directionally communicate with each other and multicast phrase packets through a shared unique channel. A CosTune user must always be free to join or leave a segment at any time. For example, in Figure 3, a CosTune user has just left segment A while another CosTune user is about to join segment B. In addition, CosTune should not be involved in multiple segments at the same time. This is because a user usually finds it hard to play and/or to listen to multiple performances simultaneously. Therefore, a segment should be exclusive.

The communications areas of CosTune and server should be comparatively small. A 20 30-m-radius area is suitable for a CosTune while a 50 100-m-radius area is suitable for a server. Larger CosTune areas, in particular, make it very difficult to find who the co-players are when the CosTune user has a jam session. Since the users may dance in performing, occlusion-free and non-directional communication is necessary.

\section{Applications and protocols}

With only one CosTune, a user can individually enjoy musical performance by herself/himself in walking on a street or in dancing. However, using the ad hoc network function together, the following two novel ways of musical activities can be achieved: ad hoc session and ad hoc collaborative composition. In this section, we describe how these two ways of musical activities are achieved with the proposed system and show skeletons of protocols to exchange phrase packets among CosTunes and servers.

\subsection{Ad hoc session}

This function brings a real-time and live musical entertainment that is one of the very important pleasures of playing music. One who wants to play a session looks for partners by strolling in a town while using this function. When the wireless communication areas of some CosTunes are overlapped, if the users' musical preferences are similar, and if all of them wants to have a session, an ad hoc real-time musical session can be immediately started there even if they meet for the first time. Needless to say, an existing band (the members of the band already knows each other) can form a street performance with this function, too.

The skeleton of the protocol for this function, as viewed from the user side, is as follows:

1. The CosTune user inputs his/her profiles and musical preferences to the CosTune beforehand.

2. The user also inputs preference information on session that the user wants to form, i.e., the music genre, the desired ensemble part and the kind of musical instrument.

3. The user wearing the CosTune travels about hunting for other CosTunes that want to form or are forming musical sessions in the desired musical genre.

4. The CosTune finds suitable partners by exchanging the user profile and session preference data.

5. If the partner candidates are already forming a session, the user listens to the performance, decides whether he/she wants to join the session, and then takes part in the session if desired.

6. If the partner candidates have not started a session yet, the user negotiate a song to be played together, and newly forms a session

If only a small number of players join the session, the sequencer of the CosTunes supplements the lacking parts with minus- $\mathrm{N}$ data that is generated based on the phrase data stored in the phrase storage or that is downloaded from a server.

This ad hoc session function enables many and unspecific people, who have the same objective, to meet each other. That is, the system can solve the conventional problem of having to find people of similar taste in order to form a musical session.

\subsection{Ad hoc collaborative composition}

This application allows people to collaborate with 
strangers who share a similar musical preference beyond time restriction. Stored phrases in the phrase storage of a CosTune can be exchanged with other CosTunes and/or servers as phrase packets when the communication areas of the CosTunes and/or the server are overlapped. However, phrase packets are not indiscriminately exchanged with any CosTunes and any servers. Referring to the owner's profile that is attached to the phrase packets, the CosTune selects partners to exchange phrase packets. Although a server can receive any phrase packets, the received phrases are classified based on the attached owner's profile data and attribute data, and are stored in the phrase storage.

The skeleton of phrase exchange protocol, as viewed from the user side, is as follows. This protocol controls phrase exchanges between CosTunes, but the protocol for phrase exchanges between a CosTune and a server can also be designed in a similar way.

1. The CosTune user inputs his/her profiles and musical preferences to the CosTune beforehand.

2. The user selects an ensemble part and a kind of musical instrument, plays a series of sound by manipulating a musical interface, and saves the sounds as a phrase with its attribute data together. Multiple phrases can be saved.

3. The user who wearing the CosTune can travel here and there hunting for other CosTunes or servers. The CosTune searches for other CosTunes or servers in its wireless communication area.

4. When the CosTune finds another CosTune, it gets the user profile data from the target CosTune, and checks for conformable conditions between the phrase owners. If the conformable conditions are satisfied, it receives a set of phrase packets whose attributes are suitable for the user's musical preference.

5. The received phrases are temporarily and sequentially stored in the phrase storage.

6. At the same time, the received phrases can be immediately played back, if the user wishes. Thus, the user can hear a music that is automatically delivered.

7. The user can select whether she/he preserves the newly obtained phrases in his/her CosTune.

8. The preserved phrases can be exchanged at the next occasion of the exchange.

Thus, a CosTune automatically collects phrases in accordance with the owner's preference and a sequence of phrases is automatically composed only by walking and passing by some other users and servers. Depending on whom the user meets, where the user goes and which route the user traces, the generated music becomes different. By exchanging phrases with some users when passing, an ad hoc collaborative composition can be achieved. Moreover, by exchanging phrases through a server, "asynchronous" ad hoc collaboration with strangers who used to pass by the server can be achieved.

Simply playing back the composed sequence, it may become a piece. However, it is usually hard to expect that the quality of such an automatically composed piece is sufficient. Therefore, manual editing and/or some automatic intelligent composition functions are needed.

\section{Prototype system}

In order to preliminarily evaluate whether we can actually perform songs while walking and whether we can form a session with a number of players using a wireless network, we created and tested a prototype system. The prototype CosTune is equipped with all of the components described in 2.1. except for the sequencer (Figure 4). We used i-cube ${ }^{\mathrm{TM}}$ system, which is an infusion systems ${ }^{\mathrm{TM}}$ product, as the A/D converter. The i-cube system converts input analog signals into MIDI data.

On the contrary, the prototype server is equipped with a sequencer. We prepared three types of interfaces: a jacket type, pants type and glove type (Figure 5). Although any kind of sound can be assigned to any type of interface, we assigned an organ sound, drum sound and strings sound to the jacket, the pants and the glove interfaces, respectively. As wireless communications component, we used wireless LAN cards (IEEE802.11b conformance, 11 Mbps). Currently, simply encapsulated MIDI data are transmitted as UDP packets among the CosTunes and the server. Neither the "phrase packet" exchange nor the protocols for the ad hoc collaborative composition and ad hoc session have been implemented yet. The transmitting delay is less than $10 \mathrm{~ms}$. This is short enough for most of the amateur players to play without confusion.

On each CosTune, performance data are converted into MIDI data and are transmitted to the server encapsulated in UDP packets. Then the server corresponds the received data to specific timbre based on the MIDI channel (a unique MIDI channel is assigned to each CosTune) and immediately broadcasts the received data as well as accompaniment data that are generated by the sequencer of the server to all of the CosTunes. Every

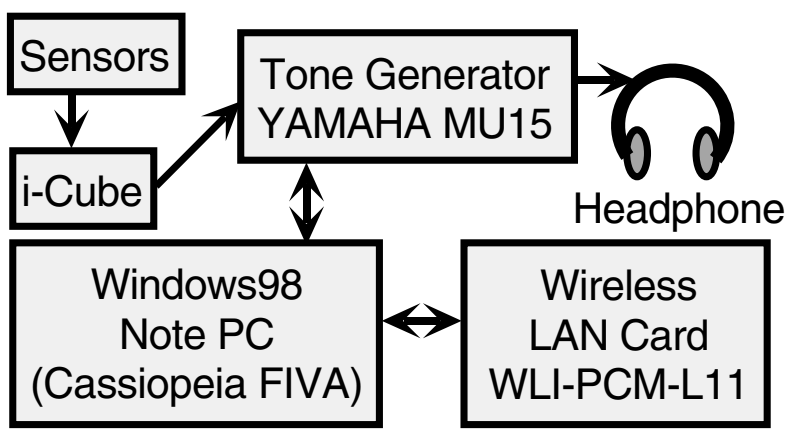

Figure 4 Construction of prototype CosTune 
CosTune receives the broadcasted data and inputs them to the tone generator. Thus, every player can listen to the performances of all of the players as well as the accompaniment through headphones.

We demonstrated a prototype system that consists of three CosTunes and one server at ATR exposition 2000 (November 1-2, 2000 in Kyoto, JAPAN) with three players for two days. We were able to comfortably hold session performances with the system while walking. Exposition visitors offered many positive comments, e.g., saying they want it immediately and that it would surely become a business. In addition, we let several visitors try a CosTune, mainly the jacket. They were able to perform quite easily while walking. Furthermore, we explained the two applications described in the previous section. Most of the visitors were deeply interested in the applications.

\section{Discussion: CosTune as a communityware in the real world}

There are several systems and projects that allow people to perform remote sessions by exchanging performance data in MIDI format over the Internet (e.g., TransMIDI [4] and RMCP [5]). CosTune is similar to these attempts in terms of phrase exchange through a network. However, as for musical performances, we regard communications in the real world as important while the communications with using the ordinary systems are done

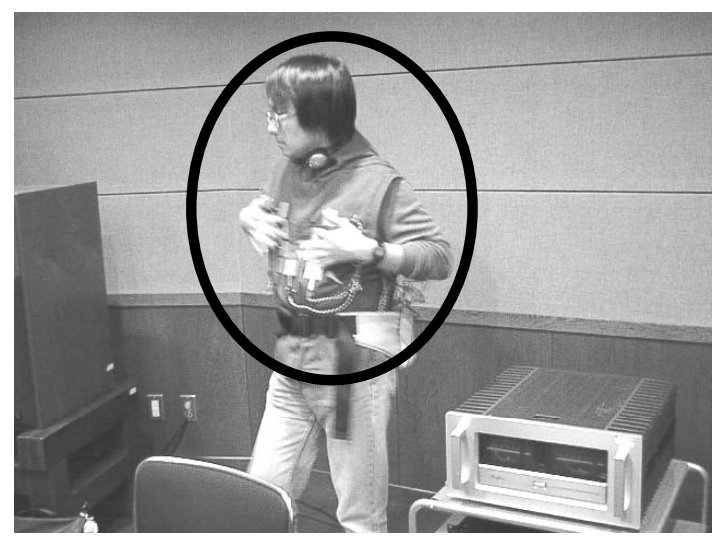

(a) Jacket type CosTune

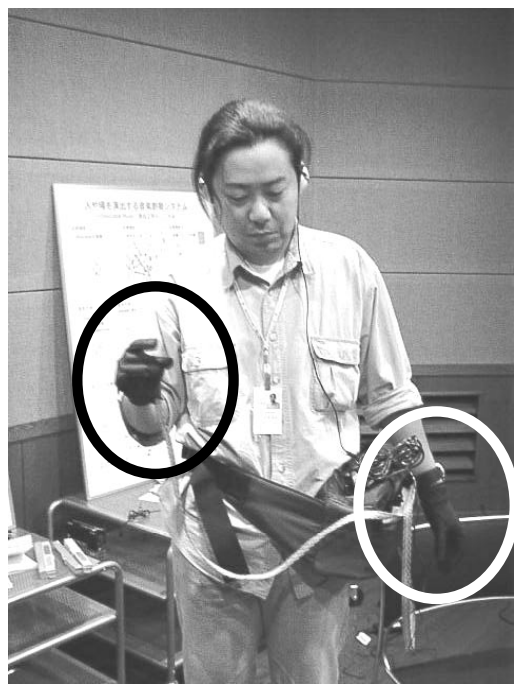

(c) Glove type CosTune

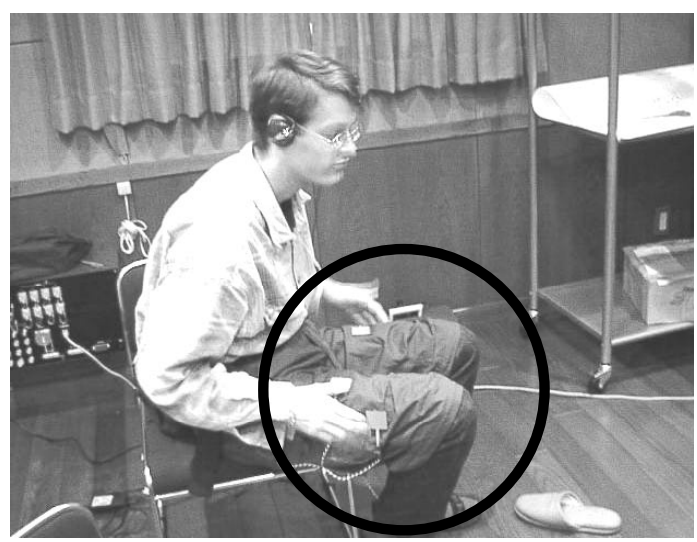

(b) Pants type CosTune

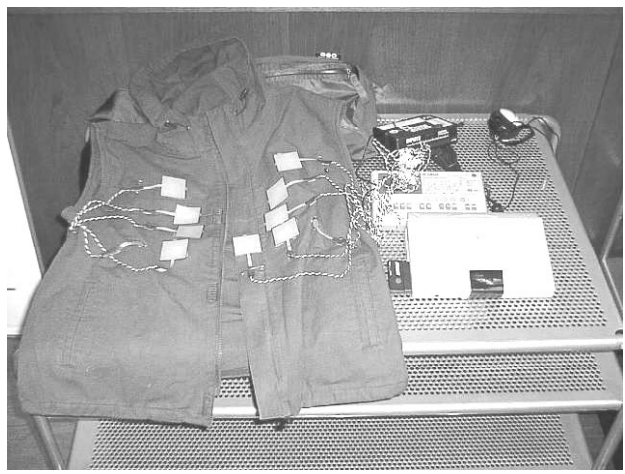

(d) All components of the jacket type CosTune

Figure 5 Prototype CosTunes 
in virtual worlds. Therefore, we required the wireless communication component that has a comparatively narrow communications area. Additionally, we required the wireless ad hoc networking function that always automatically looks for other users who have similar musical tastes. These allow people to meet in the real world and to have jam sessions in a face-to-face manner; an essential joy of musical performances.

Moreover, we are interested in the characteristics of "area" of city, town and so on. Specific kinds of people tend to gather in specific kinds of areas. They generate the "atmosphere of the area" and the atmosphere of the area attracts those who like the atmosphere. As a result, areas acquire unique characteristics, e.g., SoHo in N.Y. and Harajuku in Tokyo. We think that the music that is performed an area must reflect the characteristics of the area. Conversely, the jam session performances and the composed musical pieces must become different depending on the areas where a CosTune user visits. Therefore, we think people who want to enjoy the music of a specific area should actually (not virtually) visit the area and meet people of the area.

Like this, we aim at supporting the users of CosTunes to encounter people who have similar musical tastes as well as to encounter areas that have the users' favorite atmosphere. Thus, the CosTune supports formation of communities. Accordingly, the CosTune can be regarded as a "communityware" [6] in the real world.

\section{Conclusions}

In this paper, we described the design and the applications of CosTune, a music-mediated communications tool. CosTune is equipped with several sensors mounted on clothes as well as ad hoc networking functions to exchange phrase data and user profile information with other CosTunes and servers that will be located in places in towns. CosTune will allow a user to hold an ad hoc session on a street corner with other users who share similar musical tastes even if they just happen to meet there and do not know each other. Furthermore, using CosTune, a user can collaborate with others to compose new pieces with other unknown users who happen to pass by the user or by the server that the user happens to pass by. Thus, CosTune can support the formation of musical communities in the real world, which, we believe, will create a novel musical entertainment and musical culture.

Up to now, we have developed a very basic prototype system that is equipped with wearable interfaces and simple wireless networking functions. The prototype system was demonstrated at ATR exposition 2000, and exposition visitors offered many positive impressions. We plan to implement the phrase exchange protocol and the ad hoc session protocol in the near future. Additionally, we will employ a "bluetooth" ${ }^{\mathrm{TM}}$ " chip as an ad hoc networking device because it satisfies the requirements described in 2.4 .

Cellular phones will presently be equipped with a bluetooth $^{\mathrm{TM}}$ chip, a high-quality sound chip and the Java virtual machine. Therefore, our goal is to implement everything except for the interfaces and headphones in a cellular phone. Thus, by simply connecting a jacket and headphones to a cellular phone, and by downloading the data of a favorite song from the Web, if necessary, the cellular phone can be quickly transformed into a CosTune, thus becoming a form of musical communityware.

\section{Acknowledgment}

The authors would like to thank Dr. Yasuyoshi Sakai, Chairman of the board of ATR Media Integration \& Communications Research Laboratories, for giving us the opportunity to conduct our research. The authors also would like to thank the members of the Advanced System Development Center, YAMAHA Co., Ltd., for their kind support for prototyping the CosTune.

\section{References}

[1] Bromwich, M. A. and Wilson, J. A.: 'BODYCODER': A Sensor Suit and Vocal Performance Mechanism for Real-time Performance, Proc. International Computer Music Conference 1998, pp. 292-295, 1998.

[2] MIT Media Lab. Musical Jacket Project, http://www.media.mit.edu/hyperins/levis/

[3] Fels, S., Nishimoto, K. and Mase, K.: MusiKalScope: A Graphical Musical Instrument, IEEE Multimedia Magazine, July-September, pp.26-35,1998.

[4] Gang, D., Chockler, G. V., Anker, T. and Kremer, A.: TransMIDI: A System for MIDI Sessions Over the Network Using Transis, Proc. International Computer Music Conference 1997, pp. 283-286, 1997.

[5] Goto, M., Neyama, R. and Muraoka, Y.: RMCP: Remote Music Control Protocol --- Design and Applications ---, Proc. International Computer Music Conference 1997, pp.446-449, 1997.

[6] Ishida, T. (Ed.): Community Computing - Collaboration over Global Information Networks, John Wiley \& Sons, 1998. 